\title{
具有立体异构特征的叶绿素类二氢卟吩衍生物的合成
}

\author{
纪建业 $^{a}$ 夏尚文 ${ }^{b}$ 刘 洋 ${ }^{b}$ 殷军港 ${ }^{b}$ 祁彩霞 ${ }^{c}$ 王进军*,b,c \\ ( ${ }^{a}$ 通化师范学院化学系 通化 134002) \\ $\left({ }^{b}\right.$ 烟台大学化学化工学院 烟台 264005) \\ ( ${ }^{c}$ 山东省黄金工程技术研究中心(工业应用) 烟台 264005)
}

\begin{abstract}
摘要 以脱镁叶绿酸-a 甲酯为起始原料, 利用周环上的碳碳、碳氧和烯醇式复健, 通过加成、取代、缩合和空气氧化 等化学反应在色基上构建手性中心、顺反结构和阻旋位点. 经过旨在扩大立体差异的化学修饰, 对部分差向异构体进 行了有效分离, 完成一系列具有立体异构特征的叶绿素衍生物的合成, 并通过 UV, IR, ${ }^{1} \mathrm{H}$ NMR 及元素分析证实了 11 个未见报道的二氢卟吩衍生物的化学结构，同时对不同立体异构的形成及其表征进行了讨论.
\end{abstract}

关键词 叶绿素-a; 二氢卟吩; 立体异构; 分离; 化学反应

\section{Synthesis of Chlorophyllous Chlorin Derivatives Possessing Stereo-isomeric Character}

\author{
Ji, Jianye $^{a} \quad$ Xia, Shangwen $^{b} \quad$ Liu, Yang $^{b} \quad$ Yin, Jungang $^{b} \quad$ Qi, Caixia ${ }^{c}$ \\ Wang, Jinjun ${ }^{*, b, c}$ \\ ( ${ }^{a}$ Department of Chemistry, College of Teachers, Tonghua 134002) \\ ( ${ }^{b}$ College of Chemistry and Chemical Engineering, Yantai University, Yantai 264005) \\ ( ${ }^{c}$ Shandong Applied Research Centre of Gold Nanotechnology (Au-SDARC), Yantai 264005)
}

\begin{abstract}
Pheophorbide-a methyl ester was used as a starting material, and chiral center, cis-trans structure and atropisomic position were established on the chromophore making use of carbon-carbon, carbon-oxygen and enol form double bond. A series of synthesis of chlorophyllous derivatives possessing stereo-isomeric character were completed by addition, substitution, condensation and allomerization. The part of epimers were separated from each other effectively after chemical modification for expanding stereo-differences. The chemical structures of 11 unreported chlorins related to chlorophyll were characterized by elemental analysis, UV, IR and ${ }^{1} \mathrm{H}$ NMR spectra. The formations of different steric isomerism and their identifications were also discussed.

Keywords chlorophyll-a; chlorin; steric isomerism; separation; chemical reaction
\end{abstract}

叶绿素基本碳架上的多个手性碳原子充分地体现 出该类多取代四吡咯大环分子的特定空间结构, 其周环 上所连带的 $\mathrm{C}=\mathrm{C}$ 和 $\mathrm{C}=\mathrm{O}$ 等潜在手性单元则进一步地 丰富了大环化学中的非对称性特征, 利用色基中原有的 空间差异或者在碳架上构建新的手性中心是研究叶绿 素立体化学的有效切入途径, 所合成的大环立体异构体 在分子自组装、分子识别和光动力治疗 (Photodynamic therapy, 简称 PDT)等诸多方面的应用研究中均表现出 鲜明的独特性质 ${ }^{[1 \sim 3]}$. 为了拓展卟啉化学的深入研究,
合成更多具有应用前景的叶绿素类四吡咯大环化合物, 本文基于前期的研究工作 ${ }^{[47]}$, 以脱镁叶绿酸-a 甲酯为 起始原料，通过不同位点的多种化学反应，在二氢卟吩 周环上的相应位置进行官能团引入和结构修饰, 完成了 一系列差向、顺反和阻旋异构的叶绿素类二氢卟吩衍生 物的合成, 为篮选新型光动力抗肿瘤治疗药物提供了具 有立体差异特征的待选化合物.

脱镁叶绿酸-a 甲酯(简称 MPa, 1)于乙酸中的空气氧 化反应在 $13^{2}$-位上从上下两面引进羟基, 在多酸共存条

*E-mail: wjj1955@163.com

Received January 6, 2014; revised February 18, 2014; published online March 3, 2014.

Project supported by the National Natural Science Foundations of China (No. 21272048) and the Project of Shandong Applied Reaearch Centre of Gold Nanotechnology (2011).

国家自然科学基金(No. 21272048)和山东省黄金工程技术研究中心(2011 年度)资助项目。 
件下，使用发烟硝酸对所得差向异构体 2 进行亲电取代 反应, 分别分离出 $24 \%$ 的 $13^{2}(R)-13^{2}$-差基-20-硝基脱镁 叶绿酸(3a)和 17\%的非对映异构体 $13^{2}(S)$ - $13^{2}$-羟基- 20硝基脱镁叶绿酸 (3b); MPa (1) 在乙酸中回流脱去 $13^{2}$-位 酯基转化为焦脱镁叶绿酸-a 甲酯(简称 MPPa，4), 其 3位乙烯基与溴化氢的亲电加成反应给出 $3(R / S)$-二氢卟 吩醇 5 (67\%), 继续与 NBS 的㴪化反应则得到一对差向 异构体 3a(S)-20-溴代二氢卟吩醇 6a (21\%)和 3a( $R)$-20溴代二氢卟吩醇 $6 \mathbf{b}(24 \%)$. 利用硝酸铊对 MPa (1)的 3位乙烯基进行氧化, 以 $78 \%$ 的产率生成二氢卟吩醛 7 , 其 3-位甲酰甲基与盐酸着弪胺的胺缩合反应给出 $45 \%$ 的 3a(E/Z)-肜基取代的叶绿素衍生物 8 (Scheme 1).

MPPa (4)在氢氧化锂催化下的空气氧化反应可得 红紫素-5 二甲酯(9), 将其在含有甲醇钠的甲醇/二氯甲 烷溶液中放置, 则以 $49 \%$ 的产率转化成一对差向异构体 去氧红紫素-18 甲酯(10); 在碘催化下, 化合物 4 与硫氰 酸铵的相互作用在外接 $E$-环上引进了硫氰酸基和甲氧 基, 所得一对差向异构体焦脱镁叶绿酸 11 (46\%)在 $13^{2}$ 位上形成了立体差异; 以硼氢化钠对起始原料 1 的外接 E-环实施氧化, 同样也得到差向异构体混合物 $13^{2}$-差基$13^{2}$-去氧脱镁叶绿酸(12) (43\%). MPa 的 3-位乙烯基经四 氧化锇氧化和高碘酸钠裂解, 高产率地生成脱镁叶绿 酸-d 甲酯(13), 再与己基溴化镁在低温条件下进行 Grignard 反应, 选择性地在甲酰基上发生亲核加成, 以 51\%的产率生成差向异构体 1-羟已基取代的 MPa 14, 在
对甲苯磺酸催化下于苯中回流脱水, 结果得到 $3 \mathrm{a}(E)-3 \mathrm{~b}-$ 戊基焦脱镁叶绿酸-a 甲酯(15) (62\%) (Scheme 2).

除了构型异构以外，同时也尝试了具有构象异构性 质的叶绿素类二氢卟吩衍生物的合成研究. $\mathrm{MPa}$ (1)在含 有甲醇钠的吡啶溶液中进行空气氧化和外接环重排, 所 得红紫素-18 不经分离直接与水合肼发生肼解, 将所得 $N$-氨基红紫素-18 亚酰胺(16)与苯乙酮作用, 生成一对 阻旋异构体 $N$-苯甲亚氨基红紫素-18 亚酰胺甲酯(17) $(48 \%)$.

\section{1 结果与讨论}

\section{1 具有立体异构特征的叶绿素类二氢卟吩衍生物的 形成}

叶绿素-a 基本碳架的 C-D 环端向上连有 3 个手性中 心, 即均具有 $S$ 构型的 17-、18-位和外接 E-环的 $13^{2}$-碳 原子, 由于氢化吡咯子环中 $\beta$-位碳的周围没有连接活性 反应基团, 因此其立体构型在各种化学反应中基本保持 不变, 而 $13^{2}$-位则属于活泼的次甲基结构, 在酸或者碱 性条件下均能与酮羰基一起发生烯醇式异构, 从而丧失 其手性特征，反应结果也经常给出差向异构体混合

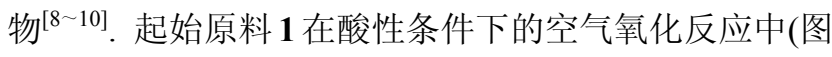
1 , 途径 a), 首先异构成烯醇异构体 $\mathbf{A}$, 再与空气中氧分 子发生作用给出过氧化物 $\mathbf{B}$, 同时在 $13^{2}$-位上形成手性 中心. 继续与质子化乙酸反应而转化为中间体 $\mathbf{C}$, 经电 子转移、羟基迁移和离去过氧乙酸则生成 $13^{2}(R / S)-13^{2}$ -

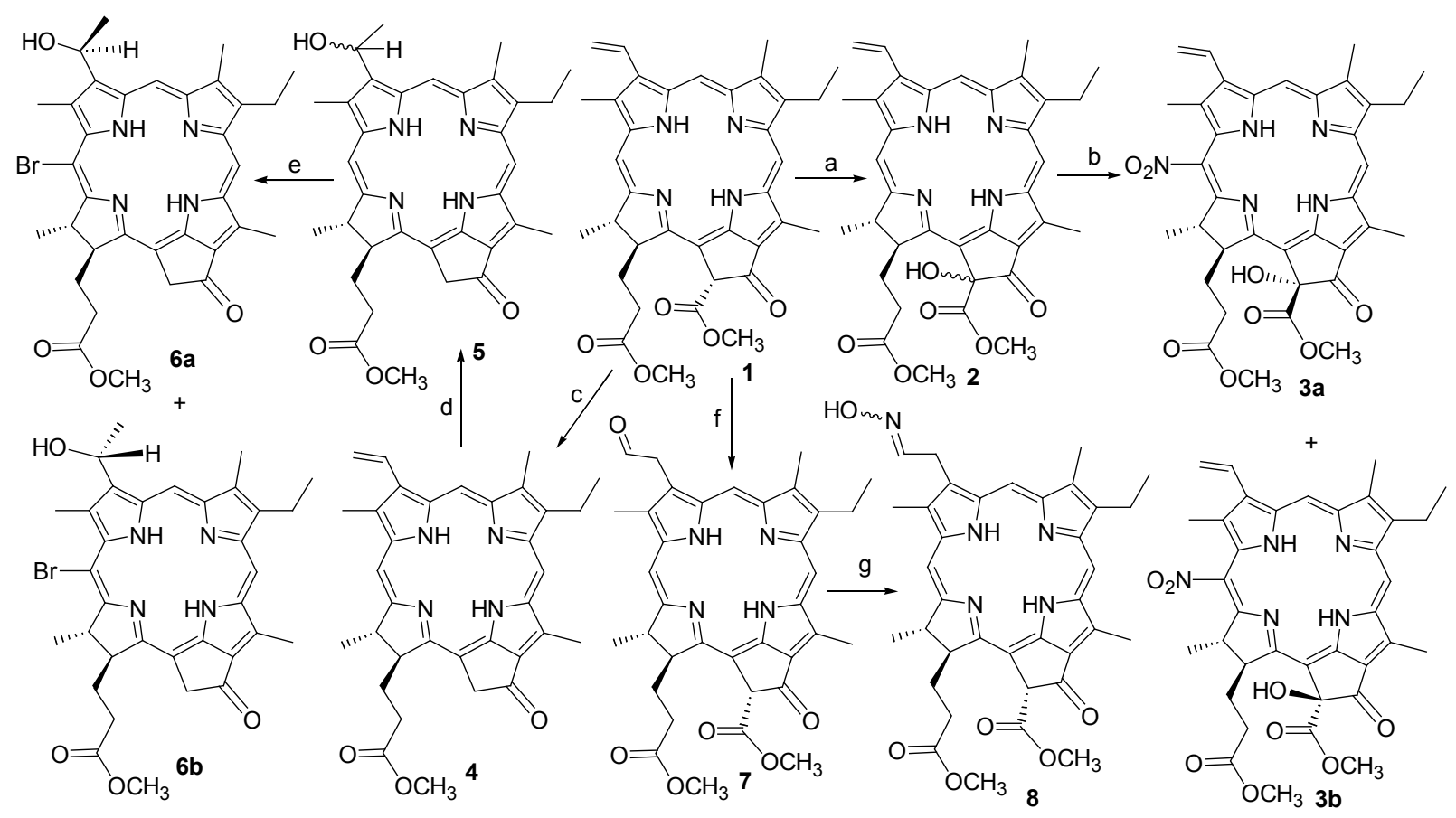

Reagents and conditions: (a) $\mathrm{AcOH} / \mathrm{O}_{2} / 110{ }^{\circ} \mathrm{C}$; (b) $\mathrm{HNO}_{3} / \mathrm{H}_{2} \mathrm{SO}_{4} / \mathrm{AcOH}$; (c) $\mathrm{AcOH} / 110{ }^{\circ} \mathrm{C}$; (d) $30 \% \mathrm{HBr}$ in $\mathrm{AcOH}$; (e) $\mathrm{NBS} / \mathrm{CH}_{2} \mathrm{Cl}{ }_{2}$; (f) $\mathrm{TI}\left(\mathrm{NO}_{3}\right)_{3} / 80 \% \mathrm{HCO}_{2} \mathrm{H} ;(\mathrm{g}) \mathrm{NH}_{2} \mathrm{OH} \mathrm{HCl} / \mathrm{Pyr} / \mathrm{CH}_{2} \mathrm{Cl}_{2}$ 


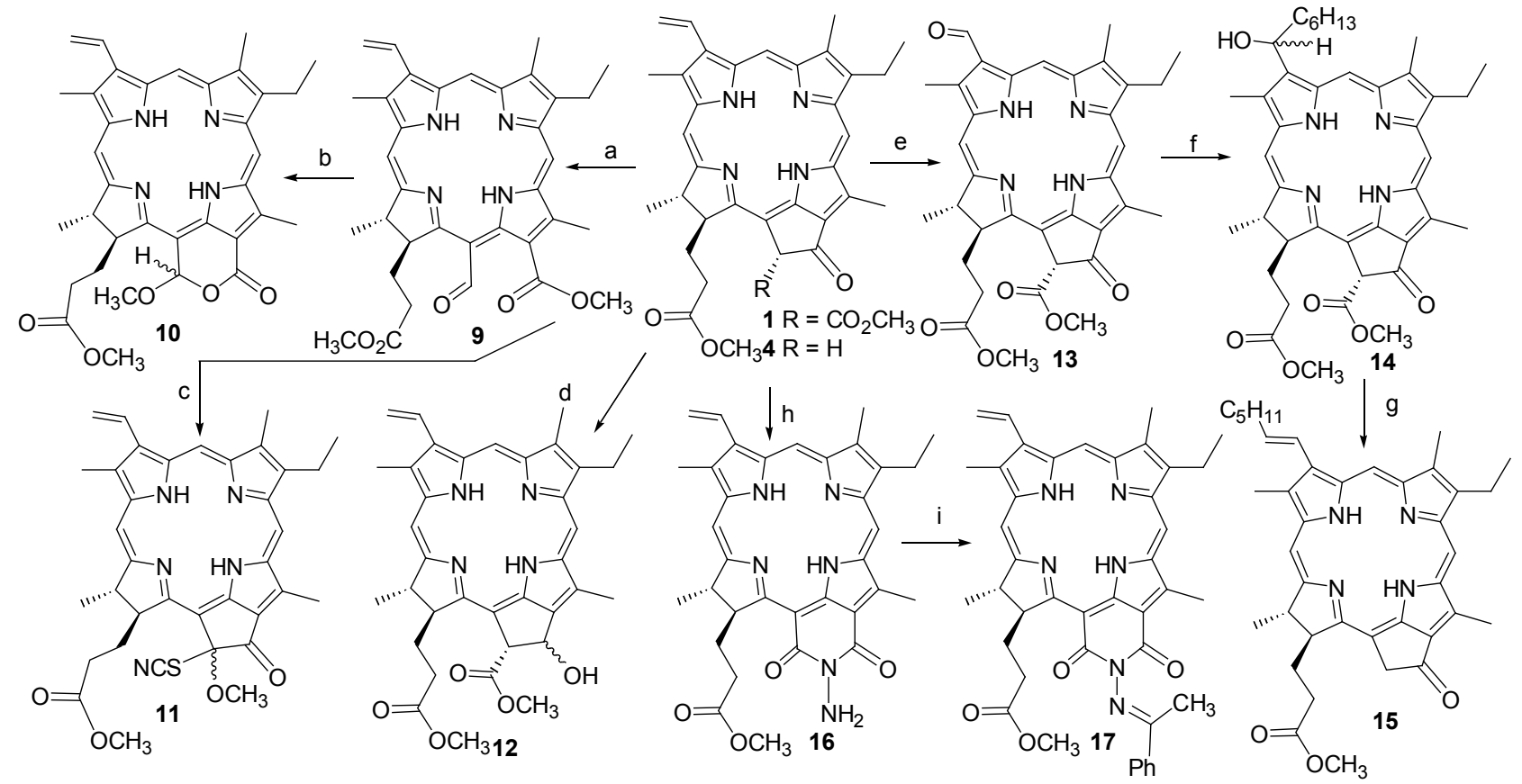

Reagents and conditions: (a) LiOH/THF/MeOH; (b) NaOMe/MOH/CH $\mathrm{N}_{2}$; (c) $\mathrm{NH}_{2} \mathrm{SCN} / \mathrm{I}_{2} / \mathrm{MeOH}$; (d) $\mathrm{NaBH} / \mathrm{MeOH}$; (e) $\mathrm{OsO}_{4} / \mathrm{NaSO}_{3} / \mathrm{NalO} \mathrm{O}_{4}$; (f) $\mathrm{C}_{6} \mathrm{H}_{13} \mathrm{MgBr} / \mathrm{THF}$; (g) $\mathrm{C}_{6} \mathrm{H}_{6} / \mathrm{TsOH} / 90{ }^{\circ} \mathrm{C}$; (h) $\mathrm{NH}_{2} \mathrm{NH}_{2} / \mathrm{CH}_{2} \mathrm{Cl}_{2}$; (i) $\mathrm{PhCOCH}_{3} /$ Toluene/120 ${ }^{\circ} \mathrm{C}$

\section{Scheme 2}

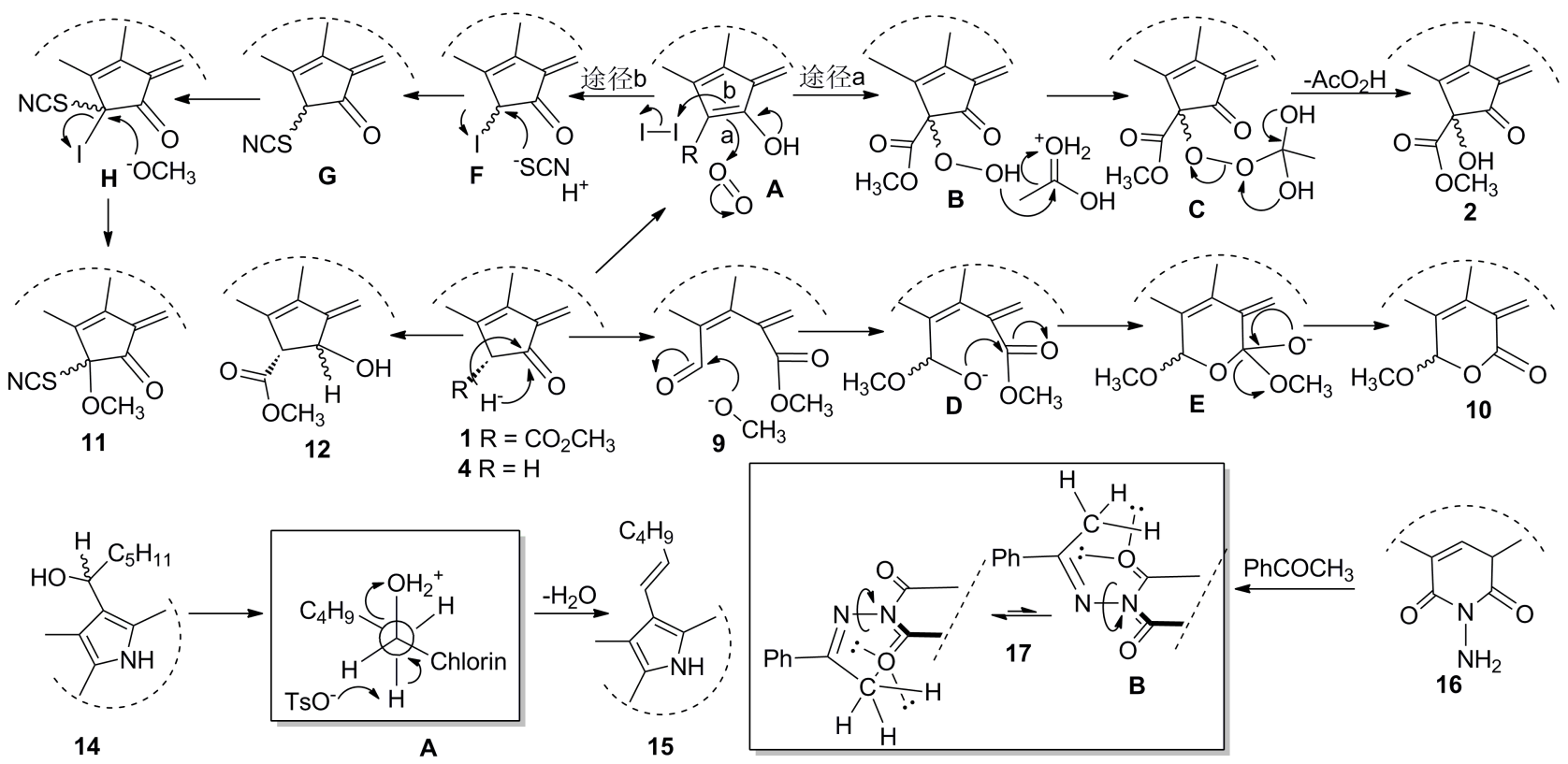

图 1 叶绿素类二氢卟吩立体异构体的形成

Figure 1 The formations of chlorophyllous chlorin steric isomers

差基脱镁叶绿酸-a 甲酯(2). MPPa (4)在氢氧化锂促进下 的空气氧化反应可以得到红紫素-5 二甲酯 $\mathbf{9}^{[11]}$, 在甲醇 钠/甲醇溶液中 15 -位甲酰基与醇氧负离子发生亲核加成 反应, 所得半缩醛 $\mathbf{D}$ 再与 13-位酯基相互作用而环合成 氢化吡喃结构, 中间体 $\mathrm{E}$ 离去一分子甲氧负离子后生成 $15 \mathrm{a}(R / S)$-15a-甲氧基-去氧红紫素-18 (10). MPPa (4)的
$13^{2}$-位亚甲基和 E-环羰基均为典型的潜在手性单元，在 碘催化与硫氰酸铵的取代反应中也是开始于外接 E-环 的烯醇式结构(途径 b), 从大环色基的上下两面先与碘 素发生反应给出差向异构混合物的碘代中间体 $\mathbf{F}$, 体系 中的硫氰酸根进攻 $13^{2}$-位碳而取代硒原子, 所形成的硫 氧酸根取代中间体 $\mathbf{G}$ 在相同条件下在 $13^{2}$-位上再次引 
进碘原子, 最后被甲氧负离子所取代生成硫氧酸根和甲 氧基双取代产物 11. 脱镁叶绿酸-a 甲酯 1 的 $13^{1}$-位羰基 的亲核加成基本都来自碳氧双键上下两个方向的进攻, 从而很容易构建新的手性中心, 当与硼氢化钠进行还原 反应时，同样在二氢卟吩醇 12 的 $13^{1}$-位上分别形成 $R$ 或者 $S$ 构型.

叶绿素降解产物在 3 位上的碳碳和碳氧双键均为潜 在的手性单元, 其加成反应的双面取向往往在周环上建 立新的手性碳结构, 例如 C(3)-烯键与溴化氢的亲电加 成和 3-位甲酰基与格氏试剂的亲核加成所形成的差向 异构体 5 和 14; 如果在周环上建立新的复健结构时, 经 常可以获得具有 $E / Z$ 异构特征的叶绿素衍生物: 文中盐 酸差弪胺与 3-甲酰甲基脱镁叶绿酸-a 甲酯(7)的肜化反应 生成一对难于分辩的 $E / Z$ 异构体 8 ; 而 $3 \mathrm{a}(R / S)$-二氢卟吩 醇 14 在脱水过程中最为优势的构象是 $3 b$-位烷基与二氢 卟吩色基处于完全交叉式(图 1 中 A 框), 因而选择性地 给出具有 $E$-式结构的 3-位取代乙烯基焦脱镁叶绿酸 15, $3 \mathrm{a}$-和 $3 \mathrm{~b}$-质子间较大的偶合常数 $(J=16.0 \mathrm{~Hz})$ 可以佐证 其立体结构.

16 与苯乙酮成腙所形成的红紫素亚酰胺衍生物 17 是一对阻旋异构体, 其立体差异主要来自亚酰胺中氮一 氮键周围的空间位阻, 由于六元酰亚胺结构中的两个碳 氧双键与环外亚胺中的所有原子均为 $\mathrm{sp}^{2}$ 杂化, 其酰亚 胺羰基上 $\mathrm{sp}^{2}$ 轨道上的非键电子在绕 $\mathrm{N}-\mathrm{N}$ 键轴旋转时 与亚胺键上所连的甲基之间将形成大面积重叠和拥阻 (图 1 中 B 框), 难于在外接环的上下两面进行自由转换, 从而形成了不能简单互变的阻旋异构现象.

\section{2 具有立体异构特征的叶绿素类二氢卟吩衍生物的 结构表征}

二氢卟吩 2 在 $13^{2}$-位上形成了新的手性中心, 由于 两个差向异构体的 E-环羟基的伸展方向正好相反, 因 而对邻近结构中的氢质子形成了不同的磁各向异性效 应, 特别是 17 -位上裂分成双重峰的单氢化学位移出现 了明显的差异, 具有 $R$-构型氧化产物 $\mathbf{a}$ 中的 17-位质子 与 $13^{2}$-位羟基处于相同一侧, 所承受的磁各向异性效应 也相对强烈, 其化学位移低场移动 $(\delta 4.69)$; 相反, $S$-构 型异构体 $\mathbf{b}$ 的 $\mathrm{C}(17)-\beta$-位氢距 $13^{2}$-位羟基的距离较远, 基本不能感受到羟氧原子中非键电子所施加的去屏蔽 效应, 因而其核磁共振信号出现在 $\delta 4.19$ 处, Dolphin 等 ${ }^{[12]}$ 曾采用高压液相再循环的方法, 完成了对立体异 构体 2 的分离及其构型确认. 为了通过更简单方法对叶 绿素类差向异构体实施分离, 我们先对混合物 $\mathbf{2}$ 进行硝 化反应, 选择性地在 20-位上引进占有较大空间的取代 基团, 扩大了差向异构体之间的立体差异, 将所得混合 物用硝酸银填充的层析柱可以清晰地将二者分离. 参比
化合物 $\mathbf{2}$ 的核磁数据, 化合物 3a 的 17-位质子吸收峰出 现在相对低场的差向异构体 $(\delta 4.70)$, 说明其与 $13^{2}$-位羟 基应该处于同侧，而核磁共振信号向高场推移的另一产 物 3b ( $\delta 4.15)$ 的相应羟基则与 $\mathrm{C}(17)-\beta$ 氢分列上下两面, 从而可确定其 $S / R$ 构型.

作为差向异构体混合物的 10 和 11 分别在外接环上 建立新的手性中心, 所连甲氧基对附近的 17-位质子也 能产生去屏蔽作用, 异构体 11 中相同位置的化学位移 之差与相互分离的 $\mathbf{3 a}$ 和 $\mathbf{3 b}$ 之间 $\Delta \delta$ 相似，而异构体 $\mathbf{1 0}$ 的 $\Delta \delta$ 相差较小, 可能由于六元环内酯中 $15 \mathrm{a}$-位甲氧基 的伸展取向距离 17- $\beta$ 位较远, 不能充分地施加磁各向 异性效应(图 2). 脱镁叶绿酸衍生物 $\mathbf{1 2}$ 的 $13^{1}$-位羟基对 $13^{2}$-位单氢同样产生去屏蔽作用, E-环上与羟基同侧的 质子振动频率相对较高, 在 $\delta=6.71$ 处出现裂分为两重 峰的吸收信号, 其偶合常数为 $6.7 \mathrm{~Hz}$, 而与羟基处于反 侧的 $\mathrm{C} 13^{1}$-位单氢的化学位移则以单峰的形式出现在相 对高场 $(\delta 6.61)$, 碳环上相邻氢原子的偶合常数与其二 面角密切相关, 处于重叠质子的偶合常数相对较大, 因 而也可作为确定大环分子立体化学的重要证据. 除此之 外, 异构体 17-位质子分别在 $\delta 4.39$ 和 4.50 出呈现处两 个裂分为双峰的单氢信号, 次要产物 $\mathbf{1 2 b}$ 的甲氧甲酰基 和羟基处于 E-环的同一侧，由于羟基的推挤，促使 $13^{2}$ 位酯基向外延展，对 17-位质子形成有效的去屏蔽作用， 从而使得其化学位移发生低场移动, 从另一侧面也间接 地对异构体的立体结构进行佐证. 18-位上的甲基和单 氢质子由于距离外接环比较远, 因而对来自于外接环的 电子效应反应并不明显，两种异构体之间的差异相对较 小, 对于同平面的羟基(或者甲氧基)的磁各向异性效应 稍有反映，与其同面的 19-位质子的化学位移向低场方 向略有移动.

焦脱镁叶绿酸-a 甲酯的 3-位乙烯基经溴化氢的亲 电加成和溴原子的进一步水解, 在 $3 \mathrm{a}$-位上建立一个新 的手性中心, Smith 等 ${ }^{[13]}$ 仍然采用 HPLC 再循环法, 艰难 地将二氢卟吩醇 5 的两个差向异构体分开. 具有 $3 \mathrm{a}(R / S)$ 异构的焦脱镁叶绿酸衍生物在核磁共振光谱中呈现出 规律性的差异，与 $S$-型异构体相比，具有 $R$-型异构体的 质子吸收相对出现在较高场, 特别是距离 3-位较近的核 磁信号 ${ }^{[12 ~ 14]}$. 同样出于在差向异构体之间产生更大空 间区别的考虑, 我们在 20 -位上引进占有较大空间结构 的溴原子，直接用硝酸银填充的层析柱，同样可以顺利 地将两个差向异构体分离, 二者的核磁共振氢谱也与同 系物的相应规律相符; 3-位连有 1-差正成基的二氢卟吩 醇 14 中两个差向异构体的氢谱信号也表现出相似的顺 序, 二者比例大约为 9:7, 作为主要产物的立体异构体 的相应化学位移均出现在较高场. 

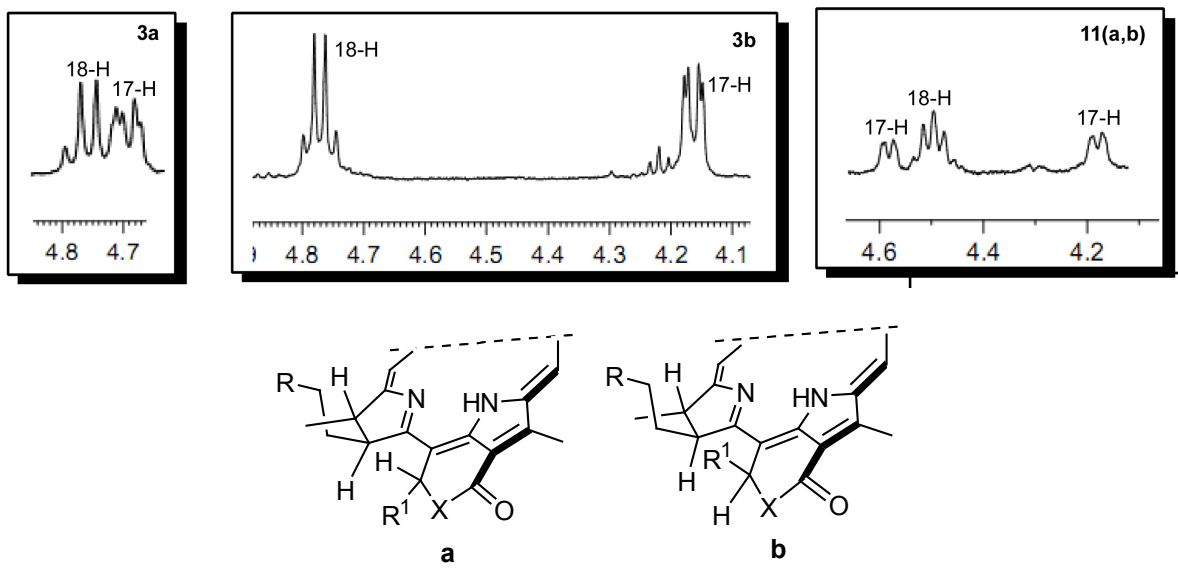

\begin{tabular}{|c|c|c|c|c|c|}
\hline \multirow{2}{*}{$X$} & \multirow{2}{*}{$\mathrm{R}^{1}$} & \multirow{2}{*}{ Compd. } & \multicolumn{3}{|c|}{$\delta$} \\
\hline & & & $17-\mathrm{H}$ & $18-\mathrm{H}$ & $18-\mathrm{CH}_{3}$ \\
\hline 0 & $\mathrm{OH}$ & $3 a$ & 4.70 & 4.82 & 1.70 \\
\hline 0 & $\mathrm{OH}$ & $3 b$ & 4.15 & 4.85 & 1.71 \\
\hline $\mathrm{O}$ & $\mathrm{OCH}_{3}$ & $10(a)$ & 4.54 & 4.47 & 1.72 \\
\hline O & $\mathrm{OCH}_{3}$ & $10(b)$ & 4.46 & 4.49 & 1.73 \\
\hline 0 & SCN & 11(a) & 4.47 & 4.48 & 1.58 \\
\hline 0 & SCN & $11 \mathrm{~b}$ & 4.17 & 4.50 & 1.58 \\
\hline
\end{tabular}

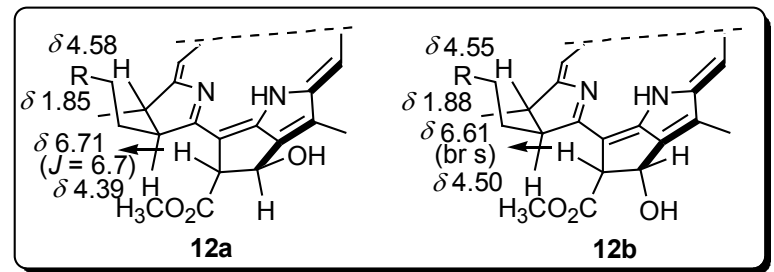

图 2 叶绿素类二氢卟吩外接环的立体结构及其附近质子的化学位移

Figure 2 The steric structure of exocyclic rings of chlorophyllous chlorins and the chemical shifts of vicinal protones

\section{2 结论}

基于叶绿素降解产物周环上的碳碳、碳氧和烯醇式 复健的反应特性，经加成、取代、缩合和空气氧化等多 种化学反应可以顺利地在大环色基上构建出手性中心、 顺反结构和阻旋位点, 进而完成一系列具有立体异构特 征的叶绿素衍生物的合成. 通过化学修饰来扩大立体异 构的空间差异, 无需 HPLC 再循环的分离手段, 采用层 析分离的经典方法, 能够简单有效地将叶绿素差向异构 体相互分离, 为合成叶绿素类二氢卟吩立体异构体及其 光物理和光生物等性质的深入研究, 提供了更为便利可 行的切入途径.

\section{3 实验部分}

\section{1 仪器与试剂}

元素分析用 Perkin-Elmer 2400 型元素分析仪测 定; IR 用 Perkin-Elmer 1730 型红外分光光度仪测定 $(\mathrm{KBr}$ 压片); UV-Vis 用 UV-160A 型紫外分光光度计测定; ${ }^{1} \mathrm{H}$ NMR 用 Brucker ARX-400 型核磁共振仪测定, 内标为
TMS. 所用试剂均为分析纯或化学纯. 脱镁叶绿酸-a 甲 酯(1)和焦脱镁叶绿酸-a (4)按文献[15]制备.

\section{$3.213^{2}(\mathrm{R} / \mathrm{S})-13^{2}$-羟基脱镁叶绿酸- $\mathrm{a}$ 甲酯(2)的合成}

将 $909 \mathrm{mg}$ (1.498 mmol)脱镁叶绿酸-a 甲酯(1)在 150 $\mathrm{mL}$ 乙酸中回流摚拌 $8 \mathrm{~h}$, 冷却后倒入 $100 \mathrm{~g}$ 碎冰中, 再 加入 $200 \mathrm{~mL}$ 二氯甲烷，分出有机层，水层用二氯甲烷 萃取 $(25 \mathrm{~mL} \times 3)$, 合并有机相, 水洗两次, 减压浓缩, 将所得浓缩物经硅胶柱层析分离[洗脱剂: $V$ (石油醚) : $V($ 乙酸乙酯 $)=3: 1$ ], 得 $625 \mathrm{mg}$ 亮绿色固体产物 2 (1.004 mmol), 产率 $67 \%$. 物理常数与分析数据与文献 [16]一致.

$3.313^{2}(R)-13^{2}$-羟基-20-硝基脱镁叶绿酸-a 甲酯(3a) 和 $13^{2}(S)-13^{2}$-羟基-20-硝基脱镁叶绿酸- $\mathrm{a}$ 甲酯(3b)的合 成

在 $10 \mathrm{~mL}$ 中三氯甲烷溶解 $113 \mathrm{mg}$ 化合物 2 (0.182 $\mathrm{mmol})$, 冷却至 $0{ }^{\circ} \mathrm{C}$, 缓慢滴加用 $0.5 \mathrm{~mL}$ 硫酸和 $2 \mathrm{~mL}$ 乙酸稀释的 $1 \mathrm{~mL}$ 发烟硝酸溶液, 撤去冰浴升温至室温, 搅拌反应 $2 \mathrm{~h}$, 先后加入 $25 \mathrm{~mL}$ 水和 $15 \mathrm{~mL}$ 三氯甲烷, 分 
出有机层, 水层用二氯甲烷萃取 $(15 \mathrm{~mL} \times 2)$, 合并有机 层, 水洗后用无水硫酸钠干燥, 减压除去溶剂, 剩余物 经硅胶柱层析分离[洗脱剂: $V$ (石油醚) $: V($ 乙酸乙酯 $)=$ $3: 1]$, 得 $68 \mathrm{mg}$ 暗红色固体产物; 将所得混合物再分别 用硝酸银填充的层析柱 $\left[m\right.$ (硅胶) $\left.: m\left(\mathrm{AgNO}_{3}\right)=100: 2\right]$, $V$ (石油醚) $： V($ 乙酸乙酯 $)=4: 1$ ]分离, 分别得到 $29 \mathrm{mg}$ 红色固体 3a $(0.044 \mathrm{mmol})$, 产率 $24 \%$ 和 $21 \mathrm{mg}$ 红色固体 3b $(0.031 \mathrm{mmol})$, 产率 $17 \%$.

3a: m.p. $224 \sim 227{ }^{\circ} \mathrm{C}$; UV-vis $\left(\mathrm{CH}_{2} \mathrm{Cl}_{2}\right) \lambda_{\max }$ (relative intensity): 417 (1.00), 517 (0.10), 548 (0.13), 618 (0.06), $678(0.49) \mathrm{nm} ;{ }^{1} \mathrm{H}$ NMR $\left(\mathrm{CDCl}_{3}\right) \delta:-1.98($ br s, $1 \mathrm{H}$, $\mathrm{NH}), 0.51(\mathrm{br} s, 1 \mathrm{H}, \mathrm{NH}), 1.57(\mathrm{~d}, J=7.1 \mathrm{~Hz}, 3 \mathrm{H}$, $\left.18-\mathrm{CH}_{3}\right), 1.70\left(\mathrm{t}, J=7.6 \mathrm{~Hz}, 3 \mathrm{H}, 8-\mathrm{CH}_{3}\right), 1.93 \sim 2.17$, $2.47 \sim 2.56$ (each m, $4 \mathrm{H}, 17 \mathrm{a}+17 \mathrm{~b}-\mathrm{H}$ ), 3.69 (q, $J=7.6 \mathrm{~Hz}$, $2 \mathrm{H}, 8 \mathrm{a}-\mathrm{H}$ ), 3.25, 3.54, 3.60, 3.62, 3.73 (each s, each $3 \mathrm{H}$, $\left.\mathrm{OCH}_{3}+\mathrm{CH}_{3}\right), 4.70(\mathrm{dd}, J=8.8,2.0 \mathrm{~Hz}, 1 \mathrm{H}, 17-\mathrm{H}), 4.82$ (q, $J=7.1 \mathrm{~Hz}, 1 \mathrm{H}, 18-\mathrm{H}), 5.35$ (s, 1H, OH), 6.16 (dd, $J=$ $17.8,1.5 \mathrm{~Hz}, 1 \mathrm{H}$, trans-3b-H), 6.29 (dd, $J=11.5,1.5 \mathrm{~Hz}$, 1H, cis-3b-H), 7.92 (dd, $J=17.8,11.5 \mathrm{~Hz}, 1 \mathrm{H}, 3 \mathrm{a}-\mathrm{H}$ ), 9.61, 9.60 (each s, each $1 \mathrm{H}$, meso-H); IR (KBr) $v: 3436(\mathrm{~N}-\mathrm{H})$, 2924, $2854(\mathrm{C}-\mathrm{H}), 1726 \sim 1678(\mathrm{C}=\mathrm{O}), 1622(\mathrm{C}=\mathrm{C})$, 1585 (chlorin skeleton), 1483, 1373, 1283, 1014, 912, 804 $\mathrm{cm}^{-1}$; Anal. calcd for $\mathrm{C}_{36} \mathrm{H}_{37} \mathrm{~N}_{5} \mathrm{O}_{8}$ : C 64.76, H 5.59, N 10.49; found C 64.68, H 5.51, N 10.69.

3b: m.p. $219 \sim 222{ }^{\circ} \mathrm{C}$; UV-vis $\left(\mathrm{CHCl}_{3}\right) \lambda_{\max }$ (relative intensity): 417 (1.00), 517 (0.10), 546 (0.12), 618 (0.06), $677(0.49) \mathrm{nm} ;{ }^{1} \mathrm{H}$ NMR $\left(\mathrm{CDCl}_{3}\right) \delta:-2.04($ br s, $1 \mathrm{H}$, $\mathrm{NH}), 0.50$ (br s, 1H, NH), $1.48(\mathrm{~d}, J=7.1 \mathrm{~Hz}, 3 \mathrm{H}$, $\left.18-\mathrm{CH}_{3}\right), 1.71\left(\mathrm{t}, J=7.6 \mathrm{~Hz}, 3 \mathrm{H}, 8-\mathrm{CH}_{3}\right), 2.08 \sim 2.17$, $2.27 \sim 2.36,2.58 \sim 2.67,2.78 \sim 2.86$ (each $\mathrm{m}, 4 \mathrm{H}, 17 \mathrm{a}+$ 17b-H), 3.71 (q, $J=7.6 \mathrm{~Hz}, 2 \mathrm{H}, 8 \mathrm{a}-\mathrm{H}$ ), 3.26, 3.61, 3.64, 3.67, 3.75 (each s, each $3 \mathrm{H}, \mathrm{OCH}_{3}+\mathrm{CH}_{3}$ ), 4.15 (dd, $J=$ 9.7, $2.4 \mathrm{~Hz}, 1 \mathrm{H}, 17-\mathrm{H}), 4.85$ (q, $J=7.0 \mathrm{~Hz}, 1 \mathrm{H}, 18-\mathrm{H})$, $5.59(\mathrm{~s}, 1 \mathrm{H}, \mathrm{OH}), 6.17(\mathrm{dd}, J=17.8,1.3 \mathrm{~Hz}, 1 \mathrm{H}$, trans-3b-H), 6.30 (dd, $J=11.5,1.3 \mathrm{~Hz}, 1 \mathrm{H}$, cis-3b-H), 7.94 (dd, $J=17.8,11.5 \mathrm{~Hz}, 1 \mathrm{H}, 3 \mathrm{a}-\mathrm{H}$ ), 9.64, 9.63 (each s, each $1 \mathrm{H}$, meso-H); IR (KBr) v: $3452(\mathrm{~N}-\mathrm{H}), 2957(\mathrm{C}-\mathrm{H})$, $1730 \sim 1680(\mathrm{C}=\mathrm{O}), 1608(\mathrm{C}=\mathrm{C}), 1520$ (chlorin skeleton), 1434, 1363, 1256, 1089, 989, $908 \mathrm{~cm}^{-1}$. Anal. calcd for $\mathrm{C}_{36} \mathrm{H}_{37} \mathrm{~N}_{5} \mathrm{O}_{8}$ : C 64.76, H 5.59, N 10.49; found C 64.88, H 5.39, N 10.27.

\section{$3.33 a(R)$-3-(1-羟基乙基)-20-溴-焦脱镁叶绿酸-a 甲 酯(6a)和 3a(S)-3-(1-羟基乙基)-20-溴-焦脱镁叶绿酸-a 甲酯的(6b)合成}

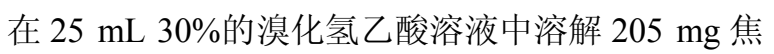

脱镁叶绿酸-a 甲酯(4) (0.374 mmol), 室温摚拌反应 $16 \mathrm{~h}$, 将反应混合物倒进 $120 \mathrm{~g}$ 碎冰中, 先后加入 $55 \mathrm{~mL}$ 二氯 甲烷和 $40 \mathrm{~mL}$ 水, 分出有机层, 水层用二氯甲烷萃取 (25 $\mathrm{mL} \times 3)$, 合并有机层, 水洗后用无水硫酸钠干燥, 减压 除去溶剂. 将所得混合物重新溶解于 $15 \mathrm{~mL}$ 二氯甲烷 中, 室温摚拌下分批加入 1.5 倍摩尔量的 $N$-溴代丁二 酰亚胺(NBS), TLC 监测, 大约 $5 \mathrm{~h}$ 后反应结束. 将反应 混合物倒入 $20 \mathrm{~mL}$ 冷水中, 分出有机层, 水层用二氯甲 烷萃取 $(20 \mathrm{~mL} \times 2)$, 合并有机层, 依次用水、碳酸氢钠饱 和溶液和水洗涤, 减压浓缩后经硅胶柱层析分离[洗脱 剂: $V$ (石油醚) $: V$ (乙酸乙酯) $=5 ： 1$ ], 得 $142 \mathrm{mg}$ 红色产 物; 将所得差向异构体混合物再用硝酸银填充的层析柱 $\left[m\right.$ (硅胶) $: m\left(\mathrm{AgNO}_{3}\right)=100: 2$, 洗脱剂: $V$ (石油醚)： $V($ 乙酸乙酯 $)=5: 1$ ]分离, 分别得到 $51 \mathrm{mg}$ 红色固体 $\mathbf{6 a}$ $(0.079 \mathrm{mmol})$, 产率 $21 \%$ 和 $58 \mathrm{mg}$ 红色固体 $6 \mathbf{b}(0.090$ $\mathrm{mmol})$, 产率 $24 \%$.

6a: m.p. 208 $211{ }^{\circ} \mathrm{C}$; UV-vis $\left(\mathrm{CH}_{2} \mathrm{Cl}_{2}\right) \lambda_{\max }$ (relative intensity): 413 (1.00), 478 (0.12), 537 (0.13), 616 (0.06), $673(0.40) \mathrm{nm} ;{ }^{1} \mathrm{H}$ NMR $\left(\mathrm{CDCl}_{3}\right) \delta$ : $-2.10($ br s, $1 \mathrm{H}$, $\mathrm{NH}), 0.51$ (br s, $1 \mathrm{H}, \mathrm{NH}), 1.45(\mathrm{~d}, J=7.2 \mathrm{~Hz}, 3 \mathrm{H}$, $\left.18-\mathrm{CH}_{3}\right), 1.69$ (t, $\left.J=7.6 \mathrm{~Hz}, 3 \mathrm{H}, 8-\mathrm{CH}_{3}\right), 2.11$ (d, $J=6.6$ $\mathrm{Hz}, 3 \mathrm{H}, 3 \mathrm{~b}-\mathrm{H}$ ), $2.12 \sim 2.39,2.41 \sim 2.64$ (each $\mathrm{m}, 4 \mathrm{H}$, $17 \mathrm{a}+17 \mathrm{~b}-\mathrm{H}$ ), 3.18, 3.23, 3.54, 3.59 (each s, each $3 \mathrm{H}$, $\left.\mathrm{OCH}_{3}+\mathrm{CH}_{3}\right), 3.66(\mathrm{q}, J=7.6 \mathrm{~Hz}, 2 \mathrm{H}, 8 \mathrm{a}-\mathrm{H}), 4.18(\mathrm{dd}, J=$ $8.6,2.9 \mathrm{~Hz}, 17-\mathrm{H}), 4.75$ (q, $J=7.2 \mathrm{~Hz}, 1 \mathrm{H}, 18-\mathrm{H}), 5.07$ (s, $\left.2 \mathrm{H}, 13^{2}-\mathrm{H}\right), 6.51(\mathrm{q}, J=6.6 \mathrm{~Hz}, 1 \mathrm{H}, 3 \mathrm{a}-\mathrm{H}), 9.52,10.21$ (each s, each 1H, meso-H); IR (KBr) v: $3327(\mathrm{~N}-\mathrm{H}), 2923$ $(\mathrm{C}-\mathrm{H}), 1733 \sim 1672(\mathrm{C}=\mathrm{O}), 1602(\mathrm{C}=\mathrm{C}), 1517$ (chlorin skeleton), 1433, 1344, 1292, 1166, 1084, 989, $925 \mathrm{~cm}^{-1}$. Anal. calcd for $\mathrm{C}_{34} \mathrm{H}_{37} \mathrm{BrN}_{4} \mathrm{O}_{4}$ : C 63.25, H 5.78, N 8.68; found $\mathrm{C} 63.38, \mathrm{H} 5.60, \mathrm{~N} 8.57$.

6b: m.p. $206 \sim 209{ }^{\circ} \mathrm{C}$; UV-vis $\left(\mathrm{CH}_{2} \mathrm{Cl}_{2}\right) \lambda_{\max }$ (relative intensity): 414 (1.00), 483 (0.08), 549 (0.14), 615 (0.08), $672(0.12) \mathrm{nm} ;{ }^{1} \mathrm{H}$ NMR $\left(\mathrm{CDCl}_{3}\right) \delta:-2.19$ (br s, $1 \mathrm{H}$, $\mathrm{NH}), 0.30$ (br s, 1H, NH), $1.38(\mathrm{~d}, J=7.1 \mathrm{~Hz}, 3 \mathrm{H}$, $\left.18-\mathrm{CH}_{3}\right), 1.66\left(\mathrm{t}, J=7.6 \mathrm{~Hz}, 3 \mathrm{H}, 8-\mathrm{CH}_{3}\right), 2.20$ (d, $J=6.6$ $\mathrm{Hz}, 3 \mathrm{H}, 3 \mathrm{~b}-\mathrm{H}), 2.02 \sim 2.13,2.23 \sim 2.32,2.36 \sim 2.46$, 2.61 2.69 (each m, 4H, 17a+17b-H), 3.19, 3.26, 3.57, 3.59 (each s, each $3 \mathrm{H}, \mathrm{OCH}_{3}+\mathrm{CH}_{3}$ ), 3.74 (q, $J=7.6 \mathrm{~Hz}$, 2H, 8a-H), 4.08 (dd, $J=8.6,2.9 \mathrm{~Hz}, 1 \mathrm{H}, 17-\mathrm{H}), 4.72$ (q, $J=7.2 \mathrm{~Hz}, 1 \mathrm{H}, 18-\mathrm{H}), 4.91\left(\mathrm{~d}, J=18.5 \mathrm{~Hz}, 1 \mathrm{H}, 13^{2}-\mathrm{H}\right)$, $5.05\left(\mathrm{~d}, J=18.5 \mathrm{~Hz}, 1 \mathrm{H}, 13^{2}-\mathrm{H}\right), 6.42(\mathrm{q}, J=6.6 \mathrm{~Hz}$, 3a-H), 9.38, 10.21 (each s, each 1H, meso-H); IR (KBr) $v$ : $3438(\mathrm{~N}-\mathrm{H}), 2923,2854(\mathrm{C}-\mathrm{H}), 1739-1680(\mathrm{C}=\mathrm{O})$, $1620(\mathrm{C}=\mathrm{C}), 1500$ (chlorin skeleton), 1452, 1365, 1222, 
1172, 1027, 981, $906 \mathrm{~cm}^{-1}$. Anal. calcd for $\mathrm{C}_{34} \mathrm{H}_{37} \mathrm{BrN}_{4} \mathrm{O}_{4}$ : C 63.25, H 5.78, N 8.68; found C 63.29, H 5.88, N 8.50.

\subsection{3 -甲酰甲基脱镁叶绿酸- $\mathrm{a}$ 甲酯(7)的合成}

在 $50 \mathrm{~mL}$ 二氯甲烷和 $25 \mathrm{~mL}$ 甲醇混合液中溶解 200 $\operatorname{mg} \mathrm{MPa}$ (1) (0.330 mmol), 在 $0{ }^{\circ} \mathrm{C}$ 下将溶解于 $30 \mathrm{~mL}$ 甲 醇的 $150 \mathrm{mg}$ 三水硝酸铊迅速加入到反应液中. 在此温 度下搅拌反应 $15 \mathrm{~min}$, 然后加入 $30 \mathrm{~mL}$ 亚硫酸氢钠甲醇 饱和溶液, 室温下搅拌 $20 \mathrm{~min}$ 后滴加 $2 \mathrm{~mL}$ 浓盐酸酸化 反应液, 有大量白色沉淀生成, 过滤除去所生成的沉淀, 加水和二氯甲烷萃取, 分出有机层水洗, 干燥后减压浓 缩. 将所得混合物溶解于 $83 \mathrm{~mL} 80 \%$ 的甲酸水溶液中, 室温摚拌 $2 \mathrm{~h}$, 加 $40 \mathrm{~mL}$ 水和 $100 \mathrm{~mL}$ 二氯甲烷萃取, 分 出水层, 有机层用饱和碳酸氢钠溶液洗涤, 再水洗一次, 减压浓缩后经硅胶柱层析分离[洗脱剂: $V$ (石油醚)： $V$ (乙酸乙酯 $)=4 ： 1]$, 得 $160 \mathrm{mg}$ 红色固体产物 $7(0.257$ $\mathrm{mmol})$, 产率为 $78 \%$. 物理常数与分析数据与文献 $[17]$ 一 致.

\section{5 (E/Z)-3-肜甲酰基脱镁叶绿酸-a 甲酯(8)的合成}

将 $70 \mathrm{mg} 7$ (0.112 mmol)溶于 $10 \mathrm{~mL}$ 甲醇中, 加入溶 于 $10 \mathrm{~mL}$ 甲醇的 $140 \mathrm{mg}$ 盐酸着弪胺, 加入 $0.1 \mathrm{~mL}$ 浓盐酸, 室温搅拌 $1 \mathrm{~h}$, 先后加 $20 \mathrm{~mL}$ 二氯甲烷和 $20 \mathrm{~mL}$ 水, 分出 有机层, 水层用二氯甲烷萃取 $(20 \mathrm{~mL} \times 2)$, 合并有机层, 依次用水、碳酸氢钠饱和溶液和水洗涤, 减压浓缩后经 硅胶柱层析分离 [洗脱剂: $V$ (石油醚) $: V$ ( 乙酸乙酯 $)=$ $3: 1$ ], 得 $32 \mathrm{mg}$ 红色固体产物 $(0.051 \mathrm{mmol})$, 产率 $45 \%$. m.p. $224 \sim 226{ }^{\circ} \mathrm{C}$; UV-vis $\left(\mathrm{CH}_{2} \mathrm{Cl}_{2}\right) \lambda_{\text {max }}$ (relative intensity): 408 (1.00), 503 (0.08), 533 (0.07), 603 (0.06), 660 $(0.38) \mathrm{nm} ;{ }^{1} \mathrm{H}$ NMR $\left(\mathrm{CDCl}_{3}\right) \delta:-1.63$ (br s, $\left.1 \mathrm{H}, \mathrm{NH}\right)$, 0.50 (br s, $1 \mathrm{H}, \mathrm{NH}$ ), 1.67 (t, $J=7.6 \mathrm{~Hz}, 3 \mathrm{H}, 8-\mathrm{CH}_{3}$ ), 1.80 (d, $\left.J=7.2 \mathrm{~Hz}, 3 \mathrm{H}, 18-\mathrm{CH}_{3}\right), 2.15 \sim 2.36,2.47 \sim 2.57$, $2.58 \sim 2.70$ (each m, 4H, 17a+17b-H), 3.19 (3.16), 3.30 (3.31), 3.58 (3.57), 3.67 (3.66), 3.88 (each s, each $3 \mathrm{H}$, $\left.\mathrm{CH}_{3}+\mathrm{OCH}_{3}\right), 3.64$ (q, $\left.J=7.6 \mathrm{~Hz}, 2 \mathrm{H}, 8 \mathrm{a}-\mathrm{H}\right), 4.20$ (d, $J=$ $8.5 \mathrm{~Hz} 1 \mathrm{H}, 17-\mathrm{H}$ ), 4.45 (q, $J=7.2 \mathrm{~Hz}, 1 \mathrm{H}, 18-\mathrm{H}$ ), (4.84) $4.68(\mathrm{~d}, J=6.3 \mathrm{~Hz}, 2 \mathrm{H}, 3 \mathrm{a}-\mathrm{H}), 6.26\left(\mathrm{~s}, 1 \mathrm{H}, 13^{2}-\mathrm{H}\right), 7.18$ (7.92) (t, $J=6.3 \mathrm{~Hz}, 3 \mathrm{~b}-\mathrm{H}), 8.12$ (7.34) (br s, $1 \mathrm{H}, 3-\mathrm{OH})$, 8.51, 9.18 (9.21), 9.48 (9.47) (each s, each $1 \mathrm{H}$, meso-H); IR (KBr) v: $3438(\mathrm{~N}-\mathrm{H}), 2923,2864(\mathrm{C}-\mathrm{H}), 1733 \sim$ $1693(\mathrm{C}=\mathrm{O}), 1610(\mathrm{C}=\mathrm{C}), 1515$ (chlorin skeleton), 1450, 1363, 1222, 1095, 908, $856 \mathrm{~cm}^{-1}$. Anal. calcd for $\mathrm{C}_{36} \mathrm{H}_{39} \mathrm{~N}_{5} \mathrm{O}_{6}$ : C 67.80, H 6.16, N 10.98; found C 67.67, H 6.10, N 10.89 .

\subsection{5a(R/S)-15a-甲氧基-15a-去氧红紫素-18 甲酯 (10)的合成}

将 $90 \mathrm{mg}$ 按照文献[11]制备的 15-甲酰基紫红素-5
甲酯(9) $(0.151 \mathrm{mmol})$ 溶于 $5 \mathrm{~mL}$ 二氯甲烷中, 再加入 15 $\mathrm{mL}$ 甲醇, 于 $0{ }^{\circ} \mathrm{C}$ 在剧烈摚拌下加入 $15 \mathrm{mg}$ 甲醇钠, 室 温搅拌 $12 \mathrm{~h}$, 先后加入 $5 \mathrm{~mL}$ 醋酸、 $20 \mathrm{~mL}$ 二氯甲烷和 $40 \mathrm{~mL}$ 水, 分出有机相后用二氯甲烷萃取水层(30 $\mathrm{mL} \times 3)$, 合并有机层，用水洗涤至中性，无水硫酸钠干 燥, 加入略超计算量的重氮甲烷振摇(约 $1 \mathrm{~min}$ ), 快速用 冰乙酸淬灭, 水洗两次, 再经无水硫酸钠干燥, 浓缩, 剩余物经硅胶柱层析分离[洗脱剂: $V$ (石油醚)：V(乙酸 乙酯 $)=3: 1$ ], 得 $44 \mathrm{mg}$ 绿色固体 $10(0.074 \mathrm{mmol})$, 产 率 49\%. m.p. 202 $205{ }^{\circ} \mathrm{C}$; UV-vis $\left(\mathrm{CH}_{2} \mathrm{Cl}_{2}\right) \lambda_{\max }$ (relative intensity): 404 (1.00), 500 (0.08), 531 (0.06), 628 (0.18), $668(0.34) \mathrm{nm} ;{ }^{1} \mathrm{H}$ NMR $\left(400 \mathrm{MHz}, \mathrm{CDCl}_{3}\right) \delta: \quad-0.80$ $(-1.13)($ br s, $1 \mathrm{H}, \mathrm{NH}),-1.37(-1.66)($ br s, $1 \mathrm{H}, \mathrm{NH})$, 1.68 (1.66) (t, $\left.J=7.6 \mathrm{~Hz}, 3 \mathrm{H}, 8 \mathrm{~b}-\mathrm{CH}_{3}\right), 1.72$ (1.73) (d, $J=$ $\left.7.3 \mathrm{~Hz}, 3 \mathrm{H}, 18-\mathrm{CH}_{3}\right), 1.95 \sim 2.18,2.37 \sim 2.56,2.58 \sim 2.79$ (each m, 4H, 17a+17b-H), 3.21 (3.19), 3.42 (3.38), 3.65 (3.74), 3.87 (3.80), 4.05 (4.00) (each s, each $3 \mathrm{H}, \mathrm{CH}_{3}+$ $\left.\mathrm{OCH}_{3}\right), 3.68$ (3.66) (q, $\left.J=7.6 \mathrm{~Hz}, 1 \mathrm{H}, 8 \mathrm{a}-\mathrm{H}\right), 4.47$ (4.49) (q, $J=7.6 \mathrm{~Hz}, 1 \mathrm{H}, 18-\mathrm{H}), 4.54(4.46)(\mathrm{d}, J=8.0 \mathrm{~Hz}, 1 \mathrm{H}$, $17-\mathrm{H}), 6.14$ (6.11) (dd, $J=11.6,1.1 \mathrm{~Hz}, 1 \mathrm{H}, c i s-3 \mathrm{~b}-\mathrm{H})$, 6.28 (6.26) (dd, $J=17.8,1.1 \mathrm{~Hz}, 1 \mathrm{H}$, trans-3b-H), 7.72 (7.48) (s, 1H, 15a-H), 7.98 (7.94) (dd, $J=17.8,11.5 \mathrm{~Hz}$, 1H, 3a-H), 9.67 (9.60), 9.49 (9.40), 8.69 (8.60) (each s, each 1H, meso-H); IR (KBr) v: $3421(\mathrm{~N}-\mathrm{H}), 2958,2925$ $(\mathrm{C}-\mathrm{H}), 1733-1691(\mathrm{C}=\mathrm{O}), 1610(\mathrm{C}=\mathrm{C}), 1542$ (chlorin skeleton), 1452, 1363, 1222, 1172, 1029, 979, $908 \mathrm{~cm}^{-1}$. Anal. calcd for $\mathrm{C}_{35} \mathrm{H}_{38} \mathrm{~N}_{4} \mathrm{O}_{5}$ : C 70.69, H 6.44, N 9.42; found C 70.80, H 6.49, N 9.59.

\section{$3.713^{2}(R / S)-13^{2}$-甲氧基- $13^{2}$-硫氰酸基焦脱镁叶绿酸- $a$ 甲酯(11)的合成}

将 $110 \mathrm{mg}$ 焦脱镁叶绿酸-a 甲酯(4) $(0.201 \mathrm{mmol})$ 溶 解于 $10 \mathrm{~mL}$ 甲醇中, 然后加入 $38 \mathrm{mg}$ 硫氰酸铵 $(0.500$ $\mathrm{mmol})$ 和 $51 \mathrm{mg}$ 碤 $(0.202 \mathrm{mmol})$, 搅拌回流反应 $4 \mathrm{~h}$, 冷 却后加入 $45 \mathrm{~mL}$ 二氯甲烷和 $40 \mathrm{~mL}$ 水, 分出有机层, 水 层用二氯甲烷萃取 $(15 \mathrm{~mL} \times 2)$, 合并有机层, 水洗后用 无水硫酸钠干燥, 减压除去溶剂, 剩余物经硅胶柱层析 分离[洗脱剂: $V$ (苯)：V(丙酤) $=10 ： 1$ ], 得 $59 \mathrm{mg}$ 暗红色 固体产物 11 (0.092 mmol), 产率 46\%. m.p. 195 $198{ }^{\circ} \mathrm{C}$; UV-vis $\left(\mathrm{CH}_{2} \mathrm{Cl}_{2}\right) \lambda_{\max }$ (relative intensity): 368 (0.61), 418 (1.00), 510 (0.11), 540 (0.10), 615 (0.08), 669 (0.47) nm; ${ }^{1} \mathrm{H}$ NMR $\left(\mathrm{CDCl}_{3}\right) \delta:-1.77$ (br s, NH), 0.35 (br s, $1 \mathrm{H}, \mathrm{NH}), 1.58\left(\mathrm{~d}, J=7.2 \mathrm{~Hz}, 2 \mathrm{H}, 18-\mathrm{CH}_{3}\right), 1.63$ (t, $\left.J=7.6 \mathrm{~Hz}, 3 \mathrm{H}, 8-\mathrm{CH}_{3}\right), 2.02 \sim 2.39,2.42 \sim 2.64,2.77 \sim$ 2.85 (each m, 4H, 17a+17b-H), 3.21, 3.39, 3.57 (3.54), 3.69 (3.67), 3.70 (3.72) (each s, each $3 \mathrm{H}, \mathrm{CH}_{3}+\mathrm{OCH}_{3}$ ), 
3.68 (q, $J=7.6 \mathrm{~Hz}, 2 \mathrm{H}, 8 \mathrm{a}-\mathrm{H}), 4.17$ (4.47) (d, $J=7.2 \mathrm{~Hz}$, $1 \mathrm{H}, 17-\mathrm{H}), 4.48(4.50)$ (q, $J=7.3 \mathrm{~Hz}, 1 \mathrm{H}, 18-\mathrm{H}), 6.20(\mathrm{~d}$, $J=11.5 \mathrm{~Hz}, 1 \mathrm{H}$, cis-3b-H), $6.30(\mathrm{~d}, J=17.8 \mathrm{~Hz}, 1 \mathrm{H}$, trans-3b-H), 7.97 (dd, $J=17.8,11.5 \mathrm{~Hz}, 1 \mathrm{H}, 3 \mathrm{a}-\mathrm{H}), 8.57$, 9.39 (9.38), 9.55 (9.54) (each s, each 1H, meso-H); IR KBr) $v: 3458(\mathrm{~N}-\mathrm{H}), 2956,2925(\mathrm{C}-\mathrm{H}), 1737-1697$ $(\mathrm{C}=\mathrm{O}), 1627(\mathrm{C}=\mathrm{C}) 1562$ (chlorin skeleton), 1465, 1373, $1228,1179,1033,991 \mathrm{~cm}^{-1}$. Anal. calcd for $\mathrm{C}_{36} \mathrm{H}_{37} \mathrm{~N}_{5} \mathrm{O}_{4} \mathrm{~S}$ : C 68.01, H 5.87, N 11.02; found C 68.13, H 5.98, N 10.91.

\section{$3.813^{1}(R / S)-13^{1}$-羟基-13 ${ }^{1}$-去氧脱镁叶绿酸-a 甲酯} (12)的合成

在 $10 \mathrm{~mL}$ 二氯甲烷中溶解 $280 \mathrm{mg}$ 化合物 $4(0.510$ $\mathrm{mmol}$ ), 再加入 $15 \mathrm{~mL}$ 甲醇稀释. 在 $0{ }^{\circ} \mathrm{C}$ 条件下缓慢滴 加含有 $93 \mathrm{mg}$ 硼氢化钠的 $5 \mathrm{~mL}$ 甲醇溶液, 室温避光摚 拌反应 $18 \mathrm{~h}$ 后, 先后向混合物中加入 $25 \mathrm{~mL}$ 二氯甲烷和 $40 \mathrm{~mL}$ 水, 分出有机层, 有机层经水洗涤 $(25 \mathrm{~mL} \times 2)$, 无水硫酸钠干燥, 减压除去溶剂, 剩余物经硅胶柱层析 分离[洗脱剂: $V$ (石油醚) $: V$ (乙酸乙酯 $)=3: 1$ ], 得 134 $\mathrm{mg}$ 墨绿色固体 12 (0.219 mmol), 产率 43\%. m.p. 204 $207{ }^{\circ} \mathrm{C}$; UV-vis $\left(\mathrm{CH}_{2} \mathrm{Cl}_{2}\right) \lambda_{\max }$ (relative intensity): 397 (1.00), 499 (0.09), 538 (0.06), 617 (0.08), $653(0.26) \mathrm{nm}$; ${ }^{1} \mathrm{H}$ NMR $\left(\mathrm{CDCl}_{3}\right) \delta:-1.27$ (1.29) (br s, $\left.1 \mathrm{H}, \mathrm{NH}\right),-3.24$ (3.18) (br s, each 1H, NH), 1.74 (1.73) (t, $J=7.6 \mathrm{~Hz}, 3 \mathrm{H}$, $\left.8-\mathrm{CH}_{3}\right), 1.85$ (1.88) (d, $\left.J=7.2 \mathrm{~Hz}, 3 \mathrm{H}, 18-\mathrm{CH}_{3}\right), 2.02 \sim$ 2.26, 2.23 2.32, 2.38 2.56, 2.58 2.68 (each $\mathrm{m}, 4 \mathrm{H}$, $17 \mathrm{a}+17 \mathrm{~b}-\mathrm{H}), 3.80$ (3.78) (q, $J=7.6 \mathrm{~Hz}, 2 \mathrm{H}, 8 \mathrm{a}-\mathrm{H}), 3.89$ (3.82), 3.60 (3.58), 3.53, 3.50 (3.37), 3.36 (3.10) (each s, each $\left.3 \mathrm{H}, \mathrm{OCH}_{3}+\mathrm{CH}_{3}\right), 4.39$ (4.50) (dd, $J=8.5,2.1 \mathrm{~Hz}$, $1 \mathrm{H}, 17-\mathrm{H}), 4.58$ (4.55) (qd, $J=7.4,1.4 \mathrm{~Hz}, 1 \mathrm{H}, 18-\mathrm{H})$, 5.91 (br s, $\left.0.3 \mathrm{H}, 13^{1}-\mathrm{H}\right), 6.14$ (6.13) (d, $J=11.5 \mathrm{~Hz}, 1 \mathrm{H}$, trans-3b-H), 6.31 (d, $J=17.8,1 \mathrm{H}$, cis-3b-H), 6.34 (d, $J=$ $\left.6.7 \mathrm{~Hz}, 0.7 \mathrm{H}, 13^{1}-\mathrm{H}\right), 6.61\left(\mathrm{br} \mathrm{s}, 0.3 \mathrm{H}, 13^{2}-\mathrm{H}\right), 6.71(\mathrm{~d}, J=$ $\left.6.7 \mathrm{~Hz}, 0.7 \mathrm{H}, 13^{2}-\mathrm{H}\right), 8.17(8.16)(\mathrm{dd}, J=17.8,11.5 \mathrm{~Hz}$, 1H, 3a-H), 9.85 (9.83), 9.65 (9.62), 8.92 (8.91) (each s, each $1 \mathrm{H}$, meso-H); IR $(\mathrm{KBr}) v$ : $3423(\mathrm{~N}-\mathrm{H}), 2927,2860$ $(\mathrm{C}-\mathrm{H}), 1739 \sim 1704(\mathrm{C}=\mathrm{O}), 1612(\mathrm{C}=\mathrm{C}), 1556$ (chlorin skeleton), 1453, 1373, 1259, 1176, 1066, $985 \mathrm{~cm}^{-1}$. Anal. calcd for $\mathrm{C}_{36} \mathrm{H}_{40} \mathrm{~N}_{4} \mathrm{O}_{5}$ : C 71.03, H 6.62, N 9.20; found C 71.17, H 6.67, N 9.04.

\section{$3.93 \mathrm{a}(R / S)$-3-(1-羟基戊基)脱镁叶绿酸-a 甲酯(14) 的合成}

在 $25 \mathrm{~mL}$ 四氢呋喃中溶解 $212 \mathrm{mg}$ 化合物 $\mathbf{1}$ (0.349 $\mathrm{mmol}$ ), 再加入 $0.15 \mathrm{~mL}$ 吡啶. 在剧烈搅拌下, 将溶解在 $2 \mathrm{~mL}$ 四氢呋喃中的 $52 \mathrm{mg} \mathrm{OsO}_{4}(0.205 \mathrm{mmol})$ 慢慢滴加 到 $0{ }^{\circ} \mathrm{C}$ 的反应液中, 并在此温度下持续摚伴 $30 \mathrm{~min}$. 然
后升至室温, 再搅拌反应 $2 \mathrm{~h}$, 加入 $35 \mathrm{~mL}$ 在 $50 \%$ 甲醇中 饱和的 $\mathrm{NaHSO}_{3}$ 溶液, 摚拌 $20 \mathrm{~min}$, 过滤除去 $\mathrm{OsO}_{3}$ 的红

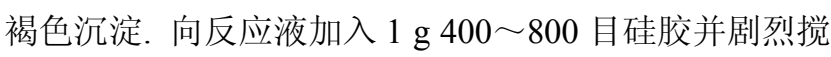
拌, 然后将溶解于 $5 \mathrm{~mL}$ 水中的 $227 \mathrm{mg} \mathrm{NaIO}$ (1.061 $\mathrm{mmol}$ )倒入反应体系, $30 \mathrm{~min}$ 后加入 $100 \mathrm{~mL}$ 水和 $80 \mathrm{~mL}$ 二氯甲烷淬灭反应, 过滤, 分出有机层, 浓缩并真空抽 干. 将初产物 13 重新溶解于 $15 \mathrm{~mL}$ 干燥的四氢呋喃中, 氮气保护, 于 $0{ }^{\circ} \mathrm{C}$ 搅拌逐滴滴加 $1 \mathrm{~mol} / \mathrm{L}$ 正已基溴化镁 乙醚溶液, TLC 跟踪反应, 约 $20 \mathrm{~min}$ 反应结束. 向反应 体系加入 $25 \mathrm{~mL}$ 的饱和氯化铵溶液, 摚拌 $20 \mathrm{~min}$ 后, 再 加入 $20 \mathrm{~mL}$ 的二氯甲烷. 分出有机层, 用二氯甲烷萃取 水相, 合并有机层并减压浓缩, 将所得剩余物经硅胶柱 层析分离[洗脱剂: $V$ (石油醚) $: V$ (乙酸乙酯 $)=4: 1$ ] , 得 到 $124 \mathrm{mg}$ 绿色固体 14 (0.178 mmol), 产率 51\%. m.p. $198 \sim 201{ }^{\circ} \mathrm{C}$; UV-vis $\left(\mathrm{CHCl}_{3}\right) \lambda_{\max }$ (relative intensity): 409 (1.00), 503 (0.16), 533 (0.15), 605 (0.14), $661(0.46)$ $\mathrm{nm} ;{ }^{1} \mathrm{H}$ NMR $\left(\mathrm{CDCl}_{3}\right) \delta:-1.87(-1.85)($ br s, $1 \mathrm{H}, \mathrm{NH})$, 0.38 (br s, $1 \mathrm{H}, \mathrm{NH}$ ), 0.81 (t, $J=6.6 \mathrm{~Hz}, 3 \mathrm{H}, 3 \mathrm{f}-\mathrm{CH}_{3}$ ), 1.69 (1.67) (t, $\left.J=7.6 \mathrm{~Hz}, 3 \mathrm{H}, 8-\mathrm{CH}_{3}\right), 1.78$ (1.77) (d, $J=7.2$ $\mathrm{Hz}, 3 \mathrm{H}, 18-\mathrm{CH}_{3}$ ), $1.56 \sim 1.80,2.11 \sim 2.52$ (each $\mathrm{m}$, all $14 \mathrm{H}, \quad 17 \mathrm{a}+17 \mathrm{~b}-\mathrm{H}+3 \mathrm{~b}-3 \mathrm{f}-\mathrm{H}), 3.31$ (3.20), 3.37 (3.34), 3.59 (3.57), 3.60, 3.87 (3.86) (each s, each $3 \mathrm{H}, \mathrm{CH}_{3}+$ $\left.\mathrm{OCH}_{3}\right), 3.68(\mathrm{q}, J=7.6 \mathrm{~Hz}, 2 \mathrm{H}, 8 \mathrm{a}-\mathrm{H}), 4.05 \sim 4.12(\mathrm{~m}, 1 \mathrm{H}$, $18-\mathrm{H}), 4.37 \sim 4.50(\mathrm{~m}, 1 \mathrm{H}, 17-\mathrm{H}), 6.07$ (q, $J=6.6 \mathrm{~Hz}, 1 \mathrm{H}$, 3a-H), 6.18 (6.14) (s, 1H, 132-H), 8.49 (8.47), 9.36, 9.59 (9.58) (each s, each $1 \mathrm{H}$, meso-H); IR (KBr) v: 3448 $(\mathrm{N}-\mathrm{H}), 2923,2854(\mathrm{C}-\mathrm{H}), 1737 \sim 1703(\mathrm{C}=\mathrm{O}), 1623$ $(\mathrm{C}=\mathrm{C}), 1504$ (chlorin skeleton), 1465, 1384, 1226, 1166, $1037 \mathrm{~cm}^{-1}$. Anal. calcd for $\mathrm{C}_{41} \mathrm{H}_{50} \mathrm{~N}_{4} \mathrm{O}_{6}: \mathrm{C} 70.87, \mathrm{H} 7.25$, N 8.06; found C 70.69, H 7.37, N 8.01.

\section{$3.10 E-3 b-$ 戊基焦脱镁叶绿酸-a 甲酯(15)的合成}

在 $25 \mathrm{~mL}$ 苯中溶解 $120 \mathrm{mg} 14$ (0.173 mmol), 再加入 $3 \mathrm{mg}$ 对甲苯磺酸, 于 $90{ }^{\circ} \mathrm{C}$ 下避光反应 $1.5 \mathrm{~h}$, 先后向反 应体系加 $25 \mathrm{~mL}$ 水和 $25 \mathrm{~mL}$ 二氯甲烷, 分出有机层并用 无水硫酸钠干燥, 减压除去溶剂, 将所得剩余物经硅胶 柱层析分离[洗脱剂: $V$ (石油醚) : $V$ (乙酸乙酯 $)=3: 1$ ], 得 $66 \mathrm{mg}$ 绿色固体 15 (0.107 mmol), 产率 62\%. m.p. $179 \sim 181{ }^{\circ} \mathrm{C}$; UV-vis $\left(\mathrm{CHCl}_{3}\right) \lambda_{\max }$ (relative intensity): 413 (1.00), 508 (0.10), 538 (0.10), 564 (0.06), 607 (0.09), $665(0.34) \mathrm{nm} ;{ }^{1} \mathrm{H}$ NMR $\left(\mathrm{CDCl}_{3}\right) \delta:-1.75($ br s, $1 \mathrm{H}$, $\mathrm{NH}), 0.46$ (br s, $1 \mathrm{H}, \mathrm{NH}$ ), 0.98 (t, $J=7.0 \mathrm{~Hz}, 3 \mathrm{H}, 3 \mathrm{f}-\mathrm{CH}_{3}$ ), $1.61\left(\mathrm{t}, J=7.6 \mathrm{~Hz}, 3 \mathrm{H}, 8 \mathrm{~b}-\mathrm{CH}_{3}\right), 1.76(\mathrm{~d}, J=7.3 \mathrm{~Hz}, 3 \mathrm{H}$, $18-\mathrm{CH}_{3}$ ), $1.40 \sim 1.75,2.11 \sim 2.37,2.40 \sim 2.78$ (each $\mathrm{m}$, $12 \mathrm{H}, 17 \mathrm{a}-\mathrm{H}+17 \mathrm{~b}+3 \mathrm{c}-\mathrm{f}-\mathrm{H}$ ), 3.14, 3.28, 3.55, 3.57 (each s, $\left.12 \mathrm{H}, \mathrm{CH}_{3}+\mathrm{OCH} 3\right), 3.68(\mathrm{q}, J=7.6 \mathrm{~Hz}, 2 \mathrm{H}, 8 \mathrm{a}-\mathrm{H}), 4.20$ 
(d, $J=8.2 \mathrm{~Hz}, 1 \mathrm{H}, 17-\mathrm{H}), 4.38$ (q, $J=7.2 \mathrm{~Hz}, 1 \mathrm{H}, 18-\mathrm{H}$ ), 5.05 (d, $\left.J=20.0 \mathrm{~Hz}, 1 \mathrm{H}, 13^{2}-\mathrm{H}\right), 5.25$ (d, $J=20.0 \mathrm{~Hz}, 1 \mathrm{H}$, $13^{2}-\mathrm{H}$ ), 6.67 (dt, $\left.J=16.0,6.9 \mathrm{~Hz}, 1 \mathrm{H}, 3 \mathrm{~b}-\mathrm{H}\right), 7.56$ (d, $J=$ $16.0 \mathrm{~Hz}, 1 \mathrm{H}, 3 \mathrm{a}-\mathrm{H}$ ), 8.43, 9.23, 9.37 (each s, each $1 \mathrm{H}$, meso-H); IR ( $\mathrm{KBr})$ v: $3443(\mathrm{~N}-\mathrm{H}), 2832(\mathrm{C}-\mathrm{H}), 1736 \sim$ $1688(\mathrm{C}=\mathrm{O}), 1653(\mathrm{C}=\mathrm{C}), 1527$ (chlorin skeleton), 1400, 1287, 1189, $793 \mathrm{~cm}^{-1}$. Anal. calcd for $\mathrm{C}_{39} \mathrm{H}_{46} \mathrm{~N}_{4} \mathrm{O}_{3}: \mathrm{C}$ 75.70, H 7.49, N 9.05; found C 75.77, H 7.33, N 9.13.

\section{$3.11 \mathrm{~N}$-氨基红紫素-18 亚酰胺甲酯(16)和 $N$-苯甲亚胺 基红紫素-18 亚酰胺甲酯(17)的合成}

在 $50 \mathrm{~mL}$ 二氯甲烷中溶解 $302 \mathrm{mg}$ 起始原料 $\mathbf{1}$ $(0.498 \mathrm{mmol})$, 再加入含有 $20 \mathrm{mg}$ 甲醇钠的 $20 \mathrm{~mL}$ 甲醇 溶液, 室温搅拌反应 $6 \mathrm{~h}$, 用 $25 \%$ 的乙酸将反应混合物 的 $\mathrm{pH}$ 值调制 3 , 并倒入 $120 \mathrm{~mL}$ 的冰水中, 再加入 $50 \mathrm{~mL}$ 二氯甲烷, 分出有机层并用无水硫酸钠干燥, 减压除去 溶剂, 将所得剩余物重新溶解于 $50 \mathrm{~mL}$ 二氯甲烷中, 氮 气保护下加入 $0.3 \mathrm{~mL} 80 \%$ 的水合联氨和 $0.2 \mathrm{~mL}$ 三乙胺, 室温搅拌反应 $4 \mathrm{~h}$, 用 $25 \%$ 的乙酸将反应混合物的 $\mathrm{pH}$ 值 调制 3 后, 将反应混合物倒进 $60 \mathrm{~mL}$ 水中, 分出的有机 层, 水洗两次, 然后用无水硫酸钠干燥, 减压除去溶剂, 将所得剩余物经硅胶柱层析分离 [洗脱剂剂: $V$ (石油 醚)：V(乙酸乙酯 $)=2: 1$ ], 得 $183 \mathrm{mg}$ 红色固体 $\mathbf{1 6}$ (0.309 mmol, 62\%). 重新将 16 溶解于 $10 \mathrm{~mL}$ 甲苯中, 再 加入 $1.5 \mathrm{~mL}$ 三乙胺和 $1.5 \mathrm{mmol}$ 量的苯乙酮, 回流反应 6 $\mathrm{h}$ 后, 倒进 $20 \mathrm{~mL}$ 的冰水中, 用二氯甲烷萃取 (20 $\mathrm{mL} \times 3)$, 水洗两次, 然后用无水硫酸钠干燥, 减压除去 溶剂, 将所得剩余物经硅胶柱层析分离[洗脱剂: $V($ 石油 醚) $: V($ 乙酸乙酯 $)=4: 1$ ], 得 $103 \mathrm{mg}$ 红色固体 17 $(0.148 \mathrm{mmol})$, 产率 $48 \%$. m.p. $235 \sim 239{ }^{\circ} \mathrm{C}$; UV-vis $\left(\mathrm{CH}_{2} \mathrm{Cl}_{2}\right) \lambda_{\max }$ (relative intensity): 381 (0.44), 436 (1.00), 530 (0.05), 569 (0.19), 708 (0.34); ${ }^{1} \mathrm{H}$ NMR $\left(\mathrm{CDCl}_{3}\right) \delta$ : $0.27(-0.21)($ br s, $1 \mathrm{H}, \mathrm{NH}),-0.12(-0.02)($ br s, $1 \mathrm{H}$, $\mathrm{NH}), 1.46$ (t, $\left.J=7.6 \mathrm{~Hz}, 3 \mathrm{H}, 8-\mathrm{CH}_{3}\right), 1.74$ (1.76) (d, $J=7.2$ $\left.\mathrm{Hz}, 3 \mathrm{H}, 18-\mathrm{CH}_{3}\right), 1.95 \sim 2.08,2.40 \sim 2.53,2.70 \sim 2.76$ ) (each m, 4H, 17a+17b-H), 2.59 (2.56), 2.79 (2.80), 3.22, 3.52 (3.58), 3.61 (3.69) (each s, each $3 \mathrm{H}, \mathrm{OCH}_{3}+\mathrm{CH}_{3}$ ), 4.33 (4.37) (d, $J=7.3 \mathrm{~Hz}, 1 \mathrm{H}, 18-\mathrm{H}), 5.26$ (5.42) (d, $J=$ $8.4 \mathrm{~Hz}, 1 \mathrm{H}, 17-\mathrm{H}), 5.98(\mathrm{~d}, J=11.5 \mathrm{~Hz}, 1 \mathrm{H}$, cis- $3 \mathrm{~b}-\mathrm{H})$, 6.08 (d, $J=17.8 \mathrm{~Hz}, 1 \mathrm{H}$, trans-3b-H), 7.61 (dd, $J=17.8$, $11.5 \mathrm{~Hz}, 1 \mathrm{H}, 3 \mathrm{a}-\mathrm{H}), 7.50 \sim 7.59$ (m, 3H, Ph-H), 8.27 (8.23) (d, $J=7.4 \mathrm{~Hz}, 2 \mathrm{H}, \mathrm{PhH}), 8.49$ (8.50), 8.89, 9.15 (9.20) (each s, each 1H, meso-H); IR (KBr) v: 3409, 3303 $(\mathrm{N}-\mathrm{H}), 2960,2929,2866(\mathrm{C}-\mathrm{H}), 1726 \sim 1689(\mathrm{C}=\mathrm{O})$, $1647(\mathrm{C}=\mathrm{C}), 1550$ (chlorin skeleton), 1460, 1380, 1271, $1205,1143,1070 \mathrm{~cm}^{-1}$. Anal. calcd for $\mathrm{C}_{42} \mathrm{H}_{42} \mathrm{~N}_{6} \mathrm{O}_{4}$ : C
72.60, H 6.09, N 12.10; found C 72.75, H 6.19, N 12.31.

\section{References}

[1] (a) Callot, H. J. In The Chlorophylls, Ed.: Scheer, H., CRC Press, Boca Raton, FL, 1991, p. 339.

(b) Mizoguchi, T.; Kim, T.-Y.; Sawamura, S.; Tamiaki, H. J. Phys. Chem. B 2008, 112, 16759 .

(c) Gryshuk, A.; Chen, Y.-H.; Goswami, L. N.; Pandey, S.; Missert, J. R.; Ohulchanskyy, T.; Potter, W.; Prasad, P. N.; Oseroff, A.; Pandey, R. K. J. Med. Chem. 2007, 50, 1754.

[2] (a) Gil, M.; Bieniaszl, M.; Seshadri, M.; Fisher, D.; Ciesielski, M. J.; Chen, Y.; Pandey, R. K.; Kozbor, D. Brit. J. Cancer 2011, 103(10), 1 .

(b) Gryshuk, A. L.; Chen, Y.-H.; Potter, W.; Ohulchansky, T.; Oseroff, A.; Pandey, R. K. J. Med. Chem. 2006, 49, 1874.

(c) Srivatsan, A.; Ethirajan, M.; Pandey, S. K.; Dubey, S.; Zheng, X.; Liu, T.-H.; Shibata, M.; Missert, J.; Morgan, J.; Pandey, R. K. Mol. Pharm. 2011, 8, 1186.

[3] (a) Gryshuk, A. L.; Graham, A.; Pandey, S. K.; Potter, W. R.; Missert, J. R.; Oseroff, A.; Dougherty1, T. J.; Pandey, R. K. Photochem. Photobiol. 2002, 76, 194.

(b) Pandey, S. K.; Gryshuk, A. L.; Sajjad, M.; Zheng, X,; Chen, Y.-H.; Abouzeid, J. M.; Charamisinau, I.; Nabi, H. A.; Oseroff, A.; Pandey, R. K. J. Med. Chem. 2005, 48, 6286.

[4] (a) Wang, J. J.; Liu, C.-L.; Li, J.-J. Synth. Commun. 2012, 42, 487. (b) Wang, L.-M.; Wang, Z.; Yang. Z.; Jin, Y.-X.; Wang, J.-J. Chin. J. Org. Chem. 2012, 32, 2154 (in Chinese).

(王鲁敏, 王振, 杨泽, 金英学, 王进军, 有机化学, 2012, 32, 2154.)

(c) Wang, J.-J.; Li, F.-G.; Li, Y.-W. Chin. J. Org. Chem. 2011, 31, 68 (in Chinese).

(王进军, 李付国, 李韵伟, 有机化学, 2011, 31, 68.)

[5] (a) Li, J.-Z.; Wang, J.-J.; Yoon, L.; Cui, B.-C.; Shim, Y.-K. Bioorg Med. Chem. Lett. 2012, 22, 1846

(b) Wang, J.-J.; Li, J.-Z.; Li, Y.-W.; Jakus, J.; Shim, Y.-K. J. Porphyrins Phthalocyanines 2010, 14, 859.

(c) Wang, J.-J.; Li, J.-Z.; Jakus, J.; Shin, K. J. Porphyrins Phthalocyanines 2012, 16, 122.

(d) Wang, J.-J.; Yin, Y.-F.; Tang, Z. J. Iran Chem. Soc. 2013, 10, 583.

[6] (a) Liu, R.-R.; Yin, J.-G.; Li, J. Z.; Wu, J.; Chen, G.-L.; Jin, Y.-X.; Wang, J.-J. Chin. J. Org. Chem. 2012, 32, 544 (in Chinese).

(刘舟冉, 殷军港, 李家柱, 武进, 金英学, 王进军, 有机化学, 2012, 32, 544.)

(b) Ji, J.Y.; Xia, S.-W.; Zhao, L.-L.; Li, J.-Z.; Qi, C.-X.; Wang, J.-J. Chin. J. Org. Chem. 2013, 33, 1457 (in Chinese).

(纪建业, 夏尚文, 赵丽丽, 李家柱, 祁彩霞, 王进军, 有机化学, 2013, 33, 1457.)

[7] (a) Wang, J. J.; Li, J.-J.; Wu, X.-R.; Shim, Y.-K. Chin. J. Chem. 2006, 24, 933.

(b) Yin, Y.-F.; Zang, Q.; Liu, Y.; Xu, X.-S.; Qi, C.-X.; Wang, J.-J. Chin. J. Org. Chem. 2013, 33, 581 (in Chinese).

(殷一焚, 张千, 刘洋, 徐希森, 祁彩霞, 王进军, 有机化学, 2013, 33, 1457.)

(c) Yin, J.-G.; Wang, Z.; Yang, Z.; Jin, Y.-X.; Wang, J.-J. Chin. J. Org. Chem. 2012, 32, 1936 (in Chinese).

(殷军港, 王振, 杨泽, 金英学, 王进军, 有机化学, 2012, 32, 1936.)

(d) Yang, Z.; Wang, Z.; Xu, X.-S.; Liu, Y.; Qi, C. X.; Wang, J.-J. Chin. J. Org. Chem. 2012, 32. 2099 (in Chinese).

(杨泽, 王振, 徐希森, 刘洋, 王进军, 有机化学, 2012, 32, 2099.) 
[8] (a) Pandey, K. R.; Goswami, L. N.; Chen, Y.-H.; Grushuk, A.; Missert, J. R.; Oseroff, A.; Dougherty, T. J. Lasers Surg. Med. 2006, 38,45 .

(b) Wang, J.-J. Chin. J. Org. Chem. 2005, 25, 1353 (in Chinese). (王进军, 有机化学, 2005, 25, 1353.)

[9] (a) Morishita, H.; Tamiaki, H. Tetrahedron 2005, 61, 6097.

(b) Kozyrev, A.; Ethirajan, M.; Chen, P.; Ohkubo, K.; Robinson, B. C.; Barkigia, K. M.; Fukuzumi, S.; Kadish, K. M.; Pandey, R. K. J. Org. Chem. 2012, 77, 10260.

[10] (a) Wang, L. M.; Wang, P.; Liu, C.; Jin, Y.-X.; Wang, J.-J. Chin. J. Org. Chem. 2012, 32, 1700 (in Chinese).

(王鲁敏, 王朋, 刘超, 金英学, 王进军, 有机化学, 2012, 32, 1700.)

(b) Wu, X.-R.; Liu, C.; Yang, Z.; Yao, N.-N.; Wang, J.-J. J. Org. Chem. 2012, 32, 632 (in Chinese).

(邬旭然, 刘超, 杨泽, 姚楠楠, 王进军, 有机化学, 2012, 32, 632.)

[11] Li, J.-Z.; Liu, W.-H.; Li, F.-G.; Wang, J.-J.; Suo, Y.-R.; Liu, Y.-J. Chin. J. Org. Chem. 2007, 27, 1594 (in Chinese).
(李家柱, 刘万卉, 李付国, 王进军, 索有瑞, 刘永军, 有机化学, 2007, 27, 1594.)

[12] Ma, L.; Dolphin, D. J. Org. Chem. 1996, 61, 2501.

[13] Smith, K. M.; Bissert, G. M. F.; Bushell, M. J. J. Org. Chem. 1980, $45,2218$.

[14] (a) Tamiaki, H.; Kitamoto, H.; Nishikawa, A.; Hibino, T.; Shibata, R. J. Org. Chem. 2004, 12, 1657.

(b) Tamiaki, H.; Komada. J.; Kunieda, M.; Fukai, K.; Yoshitomi, T.; Harada, J.; Mizoguchi, T. Photosynth. Res. 2011, 107, 133.

(c) Kunieda, M.; Yamamoto, K.; Tamiaka, H. Tetrahedron 2010, 66, 1228.

[15] Smith, K. M.; Gogg, D. A.; Simpson, D. J. J. Am. Chem. Soc. 1985, 107, 4946.

[16] Wang, J.-J.; Han, G.-F.; Wu, X.-R.; Shin, R.-J. Chin. J. Org. Chem. 2005, 25, 101 (in Chinese).

(王进军, 韩光范, 鸟旭然, 沈荣基, 有机化学, 2005, 25, 101.)

[17] Wang, P. M.S. Thesis, Yantai University, Yantai, 2009 (in Chinese).

(王朋, 硕士论文, 烟台大学, 烟台, 2009.) 